\title{
Etching and Narrowing of Graphene from the Edges
}

\author{
Xinran Wang and Hongjie Dai* \\ Department of Chemistry and Laboratory for Advanced Materials, Stanford University, \\ Stanford, CA 94305, USA \\ *Correspondence to hdai@stanford.edu
}

\begin{abstract}
:
Large scale graphene electronics desires lithographic patterning of narrow graphene nanoribbons (GNRs) for device integration. However, conventional lithography can only reliably pattern $\sim 20 \mathrm{~nm}$ wide GNR arrays limited by lithography resolution, while sub-5nm GNRs are desirable for high on/off ratio field-effect transistors (FETs) at room temperature. Here, we devised a gas phase chemical approach to etch graphene from the edges without damaging its basal plane. The reaction involved high temperature oxidation of graphene in a slightly reducing environment to afford controlled etch rate $(\leq \sim 1 \mathrm{~nm} / \mathrm{min})$. We fabricated 20-30nm wide GNR arrays lithographically, and used the gas phase etching chemistry to narrow the ribbons down to $<10 \mathrm{~nm}$. For the first time, high on/off ratio up to $\sim 10^{4}$ was achieved at room temperature for FETs built with sub-5nm wide GNR semiconductors derived from lithographic patterning and narrowing. Our controlled etching method opens up a chemical way to control the size of various graphene nano-structures beyond the capability of top-down lithography.
\end{abstract}


Graphene has attracted much attention as a novel two-dimensional system with high potential for future electronics. ${ }^{1,2}$ Narrow graphene nanoribbons (GNRs) have been demonstrated as semiconducting wires to afford field-effect transistors (FETs) with high on/off ratios at room temperature. ${ }^{3-9}$ Among various methods of producing narrow GNRs ranging from chemical sonication ${ }^{3}$ to unzipping of carbon nanotubes, ${ }^{5,10}$ top-down lithographic patterning of large pristine graphene sheet into GNRs is appealing for large-scale device integration. ${ }^{2}$ Thus far, patterning methods have only produced GNR arrays down to $\sim 20 \mathrm{~nm}$ in width (except for short, narrow constrictions ${ }^{9}$ ) limited by lithography resolution, ${ }^{7,8}$ while GNRs narrower than $\sim 5 \mathrm{~nm}$ with sufficient bandgaps are needed for room temperature FET operation..$^{3-6,11-13}$ Here, we devise a gas phase chemical approach to etch graphene from the edges without damaging the basal plane of graphene. The reaction involves high temperature oxidation of graphene in a slight reducing environment, to afford controlled ( $\leq$ $\sim 1 \mathrm{~nm} / \mathrm{min}$ ) etching of graphene. We fabricated $\sim 20-30 \mathrm{~nm}$ wide GNR arrays by electron beam lithography, and subsequently narrowed the GNRs by the gas phase etching chemistry. As the GNRs were narrowed to the $\leq \sim 5 \mathrm{~nm}$ regime, bandgap opening related to quantum confinement was clearly observed in GNR-FETs. For the first time, high on/off ratio up to $\sim 10^{4}$ was achieved at room temperature for devices of $\sim 5 \mathrm{~nm}$ wide GNRs derived from lithographic patterning. Parallel arrays of $\sim 8 \mathrm{~nm}$ wide GNRs were used to produce FETs with on/off ratio $\sim 50$ and on-currents far exceeding those of single-ribbon devices. Controlled chemical etching could play important roles in tailoring the dimensions of graphene for large scale device integration.

To devise a gas-phase chemistry for etching and narrowing graphene from the edges without creating defects in the basal plane, we investigated chemical etching of few-layer $(\leq$ 3-layer) mechanically exfoliated graphene on $300 \mathrm{~nm} \mathrm{SiO}_{2} / \mathrm{Si}$ substrate under various 
oxidation conditions at high temperatures (Fig. 1, supplementary Fig. S1\&S2. Also see Methods for experimental details). We varied the partial pressure of $\mathrm{O}_{2}$, and introduced $\mathrm{Ar}$, $\mathrm{H}_{2}$ or $\mathrm{NH}_{3}$ as dilution gas or to provide a reducing environment. We found that the etching of graphene was highly dependent on the gas mixture, reaction temperature as well as the number of layers of graphene (Table S1). At $750^{\circ} \mathrm{C}, \sim 100 \mathrm{mTorr}$ of $\mathrm{O}_{2}$ diluted by $\sim 1$ Torr of Ar or $\mathrm{H}_{2}$ gave an etch rate of $\sim 3-5 \mathrm{~nm} / \mathrm{min}(\sim 6-7 \mathrm{~nm} / \mathrm{min})$ for single (double) layer graphene (Fig. S1). Interestingly, when using $\sim 100 \mathrm{mTorr}_{2}$ with $\sim 1$ Torr $10 \% \mathrm{NH}_{3}$ in $\mathrm{Ar}$ as additive, we observed a considerably slower etching rate of $\sim 2-2.5 \mathrm{~nm} / \mathrm{min}(\sim 4 \mathrm{~nm} / \mathrm{min})$ for single (double) layer graphene (Fig. S2). We then reduced the $\mathrm{O}_{2}$ partial pressure to $\sim 25 \mathrm{mTorr}$ aimed at slower etching. Under $\sim 25 \mathrm{mT}$ Torr $\mathrm{O}_{2}$ and $\sim 1$ Torr $\mathrm{H}_{2}$, the etching rate at $800^{\circ} \mathrm{C}$ was $\sim 3-3.5 \mathrm{~nm} / \mathrm{min}(\sim 3.8-5 \mathrm{~nm} / \mathrm{min})$ for single (double) layer graphene (Fig. S1). By introducing $\mathrm{NH}_{3}$ into the system, we found that the etching rate was further decreased to less than $\sim 1 \mathrm{~nm} / \mathrm{min}$ under $\sim 25 \mathrm{mT}$ Torr of $\mathrm{O}_{2}$ in $\sim 1$ Torr of $10 \% \mathrm{NH}_{3}$ in Ar (Fig.1, S1 and Table S1). The slow etching rate was highly desirable for controlled etching and narrowing of graphene and GNRs down to the $\leq \sim 5 \mathrm{~nm}$ regime.

We observed that graphene etching occurred mostly at the edges and proceeded inward isotropically (Fig. 1, S1\&S2). Due to bond disorders and functional groups, the chemical reactivity of graphene edge carbon atoms was higher than the perfectly bonded $\mathrm{sp}^{2}$ carbon atoms in the basal plane. ${ }^{14}$ As a result, graphene sheets were etched uniformly from the edges under controlled oxidation conditions. Occasionally, we observed holes or trenches formed in graphene after reactions, due to etching initiated at point defects or line defects within graphene plane respectively (Fig.1d-f \& Fig.S1). ${ }^{14-16}$ Certain line defects were visible under atomic force microscopy (AFM) such as wrinkles (Fig. 1d), though not all defects were observed by AFM (Fig.S1a-b\&d-e). Importantly, Raman spectroscopic mapping and imaging revealed that etching from the edges did not damage the basal plane of pristine graphene, as indicated by the absence of defect related Raman band ( $\mathrm{D}$ band) in the plane of graphene sheets after etching (e.g. Fig.1g-i \& Fig.S2c-d). We observed red-shifts of the Raman G band 
of graphene after etching in both $\mathrm{O}_{2} / \mathrm{NH}_{3}$ (Fig. 1i) and $\mathrm{O}_{2} / \mathrm{Ar}$ conditions, which was not fully understood but could be due to strain in the graphene sheets as indicated by the formation of wrinkles sometimes observed after the high temperature reaction (Fig. 1e, Fig. S1b,S1e). ${ }^{17,18}$

Our graphene etching process involves $\mathrm{O}_{2}$ oxidation of graphene into $\mathrm{CO}$ or $\mathrm{CO}_{2}$. During etching, $\mathrm{O}_{2}$ molecules exothermally dissociate and form bonds with dangling carbon atoms on defect sites and edges. ${ }^{15,16}$ The slower etching rate under $\mathrm{NH}_{3}$ environment was attributed to in-situ $\mathrm{NH}_{3}$ reduction of oxygen groups formed during graphene oxidation. Our recent work on the reduction of graphene oxide suggested that $\mathrm{NH}_{3}$ was more effective than $\mathrm{H}_{2}$ in reducing oxygen groups in graphene oxide. ${ }^{19}$ The $\mathrm{NH}_{3}$ reduction effect could impede the oxidation of graphene when mixed with oxygen. Also interesting was that under the same reaction condition, we found that thicker graphene sheets were etched faster than thinner ones (Table S1, Fig. S2), similar to earlier observations made on thick graphite. ${ }^{15,16}$ This was not understood but could be due to synergistic effects of oxygen groups at the edges of adjacent layers, giving self-catalyzed etching at the edges of multi-layer graphene. ${ }^{16}$ As a control, we found that heating graphene in pure ammonia did not give etching effects (Fig.S3a).

Next, we fabricated GNR arrays by lithographic patterning, and used our gas phase reaction to narrow the as-made ribbons down to several nanometres in width. GNR arrays were fabricated by electron beam lithography (EBL) and Ar plasma etching on 300nm $\mathrm{SiO}_{2} / \mathrm{Si}$ substrate (Fig. 2a, see Methods for detailed fabrication process). Unlike previously used electron beam resist as etch mask, ${ }^{7-9}$ we used thin $(\sim 6.5 \mathrm{~nm})$ Al lines (defined by singlepixel electron beam) on exfoliated graphene as etch mask (Fig. 2a and supplementary information). The widths of GNRs were measured by AFM with finite tip size deconvolution. ${ }^{3}$ The as-patterned GNRs were down to $\sim 20 \mathrm{~nm}$ in width, with a mean edge roughness $\delta w=\left(w_{\max }-w_{\min }\right) / 2 \leq \sim 5 \mathrm{~nm}$ (Fig. 2b-g). The edge roughness was caused by random fluctuating factors in lithography and plasma etching processes. ${ }^{6-9}$ Lithographic patterning using $\mathrm{Al}$ as etch mask is versatile in patterning graphene into various structures with high reproducibility and consistency. For examples, we readily fabricated $w \sim 20 \mathrm{~nm}$ GNR 
alphabetic characters and junctions (Fig. 2d-g), which could be interesting structures to study electron transport along various crystallographic directions of graphene. ${ }^{20,21}$

To afford GNR-FETs with high $I_{o n} / I_{\text {off }}$ ratio at room temperature, $w \leq \sim 5 \mathrm{~nm}$ GNRs with sufficient bandgap $\left(E_{g}>>k_{B} T \sim 26 \mathrm{meV}\right)$ are needed..$^{3-6,11-13}$ We narrowed down the asmade $w \sim 20 \mathrm{~nm}$ GNRs using the $0.5-1 \mathrm{~nm} / \mathrm{min}$ etching condition $\left(25 \mathrm{mTorr} \mathrm{O}_{2}\right.$ in 1 Torr $\mathrm{NH}_{3} / \mathrm{Ar}$ at $800^{\circ} \mathrm{C}$ ). It was difficult to narrow GNRs with good width control in several control experiments under other conditions (Fig. S3). We succeeded in narrowing the GNRs uniformly from $\sim 20 \mathrm{~nm}$ to $\sim 8 \mathrm{~nm}$ without obvious breaks along the ribbons (Fig. 3a\&b). However, further narrowing typically resulted in breaks due to edge roughness and width variations in as-patterned GNRs. We obtained $w \leq \sim 5 \mathrm{~nm}$ ribbons by over-etching, with most GNRs evolving into discontinuous segments down to sub-5nm in width. Some of the segments exhibited sufficiently long length useful for integration into FET devices for electrical measurements (Fig. 4d). Edge roughness of as-made GNRs by lithographic patterning is currently a limiting factor in producing long, uniform ultra-narrow GNRs over large areas.

We carried out Raman spectroscopic measurements on the as-made and narrowed GNRs (Fig. 3c\&d). For $w \sim 20 \mathrm{~nm}$ as-made GNRs, several pronounced peaks were observed, including $\mathrm{D}$ band $\left(\sim 1340 \mathrm{~cm}^{-1}\right)$, G band $\left(\sim 1590 \mathrm{~cm}^{-1}\right)$, D' band $\left(\sim 1620 \mathrm{~cm}^{-1}\right)$ and $2 \mathrm{D}$ band $\left(\sim 2670 \mathrm{~cm}^{-1}\right)$ (Fig. 3c). The intensity ratio between $\mathrm{D}$ and $\mathrm{G}$ bands $I_{D} / I_{G}$ was $\sim 1-2$. The presence of defect-related D and D' bands was attributed to the edges of GNRs since no D band was observed in the parent graphene sheet. ${ }^{5,22}$ For a $w \sim 20 \mathrm{~nm}$ GNR, about $1 \%$ carbon atoms are at the edges, resulting in D and D' bands as expected. ${ }^{22}$ We observed lower $I_{D} / I_{G}$ ratio in wider GNRs (supplementary information and Fig. S7), consistent with reduced edge effects. After narrowing below $\sim 10 \mathrm{~nm}$, the intensity of G and 2D bands of graphene reduced, $I_{D} / I_{G}$ ratio increased and the $G$ and $D^{\prime}$ peaks were broadened to form a single and up-shifted G peak (Fig.3d). ${ }^{22}$ These changes in Raman spectra were due to higher percentage of edge atoms in narrowed GNRs. The $I_{D} / I_{G}$ ratios of our narrowed GNR were larger than similar 
width GNRs derived chemically, ${ }^{4}$ suggesting higher degree of edge roughness and disorder in the former.

We fabricated electrical devices on as-made and narrowed GNRs with Pd source and drain electrodes and heavily doped Si back-gate (see Methods). At room temperature, devices of as-made $w \sim 20 \mathrm{~nm}$ GNR and GNR arrays typically showed $I_{\max } / I_{\min }$ current ratios less than $\sim 3$ (Fig. S4), ${ }^{5,6,8}$ indicating insufficient bandgap (compared to $k_{B} T \sim 26 \mathrm{meV}$ ) for room temperature FETs. The bandgap of a perfect GNR is predicted to scale inverse linearly with GNR widths, $E_{g} \sim(0.3-1.5 \mathrm{eV} \cdot \mathrm{nm}) / \mathrm{w}(\mathrm{nm})$ depending on the orientation and edge configuration of the ribbons. ${ }^{12,13}$ For GNRs narrowed down to $\sim 10 \mathrm{~nm}$, we observed an $I_{\max } / I_{\min }$ ratio $\sim 10$ at room temperature (Fig. S5). With long GNRs narrowed down to $w \sim 8 \mathrm{~nm}$ with good continuity (Fig. 3b), we fabricated a FET using an array of GNRs in parallel and inter-digitized electrodes as contacts (Fig. 4a). We observed high $I_{\text {on }} / I_{\text {off }}$ ratio up to $\sim 50$ at room temperature and on-state current of $\sim 20 \mu \mathrm{A}$ ( $\sim 40$ times that of single ribbon devices) at $V_{d s}=-500 \mathrm{mV}$ (Fig. 4b\&c) with $\sim 40$ GNR sections (channel length $\sim 160 \mathrm{~nm}$ ).

For GNRs narrowed down to the $w \leq \sim 5 \mathrm{~nm}$ regime, limited by discontinuity in the ribbons, we fabricated devices only on single ribbons rather than with parallel arrays. Figure 4e\&f show room temperature $I_{d s}-V_{g s}$ and $I_{d s}-V_{d s}$ characteristics of a GNR-FET with a $w \sim 4 \mathrm{~nm}$ GNR (see Fig. S6 for another example). The device showed ambipolar transport in air with $I_{\text {on }} / I_{\text {off }}>10^{4}$ (Fig. 4e), a clear evidence for bandgap opening by lateral quantum confinement. $^{3-6,11-13}$ This was the highest room-temperature $I_{\text {on }} / I_{\text {off }}$ ratio reported for GNRs derived from lithographic patterning. ${ }^{6-9}$ In vacuum, the device show an intrinsic n-type behaviour with threshold voltage shifted to the negative gate-voltage side due to desorption of physisorbed species including oxygen. ${ }^{23}$ Based on the ambipolar $I_{d s}-V_{g s}$ characteristics, we estimated the bandgap of the GNR to be $E_{g} \sim 0.4 \mathrm{eV}$ from $I_{o n} / I_{\text {off }} \sim \exp \left(E_{g} / 2 k_{B} T\right)$ since the offstate current was thermally activated over a body-Schottky Barrier of $\sim E_{g} / 2$ (Ref. 8). 
We developed controlled chemical narrowing of graphene to afford quantum confined structures. Parallel GNR arrays were made to afford graphene FETs with high on-currents and high on/off ratio of $\sim 50$ at room temperature. Single ribbons based on lithography were narrowed below $\sim 5 \mathrm{~nm}$ to afford on/off ratios of $\sim 10^{4}$. On the single-ribbon basis, our narrowed GNRs afforded lower on-currents than our previously reported chemically derived sub-10nm GNRs on the same $300 \mathrm{~nm} \mathrm{SiO}_{2}$ substrate. ${ }^{3}$ The narrowed lithography-derived GNRs showed rougher and more disordered edges, as reflected from Raman spectroscopy data. Edge disorder caused scattering effects that contributed to the low on-currents of GNR devices. $^{4,24-26}$ There are variations between devices such as the doping, likely due to the differences in the detailed edge structures. Clearly, the success of our approach is currently limited by the edge roughness introduced in the patterning process, and much effort should be directed in the future towards making smooth edges by either improving lithographic patterning processes, or developing novel chemical means to perfect the edges. Some recent experiments have shown the promise of making graphene and GNRs with atomically well defined edges by anisotropic etching, ${ }^{27-30}$ which could be combined with narrowing to produce long, uniform sub-5nm GNR semiconductors to produce high performance graphene transistors for potential digital applications. This could be an appealing roadmap for graphene especially since chemical narrowing of GNRs with well defined crystallographic orientations should be possible to afford semiconducting GNRs with desired edge structures.

\section{Methods}

\section{Preparation of mechanically exfoliated graphene, microscopy and spectroscopy characterizations and lithographic patterning of GNRs}

Graphene sheets used in etching experiments under various conditions were mechanically exfoliated from highly oriented pyrolitic graphite crystals on a thermally grown $\sim 300 \mathrm{~nm} \mathrm{SiO}_{2} / \mathrm{p}^{++} \mathrm{Si}$ substrate using $\mathrm{Scotch}^{\circledR}$ tape. ${ }^{31}$ A subsequent annealing in $\sim 2$ Torr $\mathrm{H}_{2}$ at 
$800^{\circ} \mathrm{C}$ for $\sim 15 \mathrm{mins}$ was done to clean the substrate and graphene sheets. Then AFM and Raman mapping were used to characterize the graphene sheets before and after the gas phase reaction. Raman mapping was done by a Horiba Jobin Yvon LabRAM HR Raman microscope with a $633 \mathrm{~nm}$ He-Ne laser excitation (spot size $\sim 1 \mu \mathrm{m}$, power $\sim 5 \mathrm{~mW}$ ). We used $100 \mathrm{~nm}$ step size and 4 seconds accumulation time to map the graphene sheets.

Graphene sheets used for patterning GNRs were mechanically exfoliated on a thermally grown $\sim 300 \mathrm{~nm} \mathrm{SiO}_{2} / \mathrm{p}^{++} \mathrm{Si}$ substrate with pre-patterned $\mathrm{Ti} / \mathrm{Au}$ markers and were annealed at $\sim 600^{\circ} \mathrm{C}$ for $\sim 15 \mathrm{mins}$ (Ti/Au markers melt at higher temperature). We used optical microscope to locate few-layer ( $\leq 3$-layer) graphene sheets and then did Raman spectroscopy to determine the number of layers. We spun $\sim 70 \mathrm{~nm}$ PMMA with molecular weight of 950kDa as EBL resist. Single pixel lines were exposed in a Raith 150 system in the Stanford Nanofabrication Facility with acceleration voltage of $10 \mathrm{keV}$ and line dosage of $650 \mu \mathrm{C} / \mathrm{cm}$. Wider GNRs could be made by exposing areas instead of single pixel lines. The development was done in cold $\left(4^{\circ} \mathrm{C}\right)$ 1:3 methyl iso-butyl ketone: isopropanol solution for 75 seconds to afford good edge roughness in the resist profile. ${ }^{32}$ A $6.5 \mathrm{~nm}$ thick Al film was then electron beam evaporated, followed by standard liftoff. The Ar plasma etching was done in a MRC plasma etcher for $20-30$ s (depending on number of layers of graphene), under an Ar flow rate of $\sim 10 \mathrm{~cm}^{3} / \mathrm{min}$, chamber pressure $\sim 40 \mathrm{mTorr}$ and plasma power $\sim 10 \mathrm{~W}$. After plasma etching, the chips were soaked in $0.1 \mathrm{~mol} / \mathrm{L} \mathrm{KOH}$ water solution for $\sim 2$ minutes to remove the Al lines. We then annealed the chips in $\sim 2 \mathrm{Torr} \mathrm{H}_{2}$ at $600^{\circ} \mathrm{C}$ to clean resist residues from the substrate.

\section{Gas phase chemical etching of graphene in a vacuum furnace}

Gas phase etching of graphene sheets and narrowing of GNRs were carried out in a vacuum furnace connected to a mechanical pump, with a base pressure of $\sim 15 \mathrm{mT}$ Trr. Note that a leakfree vacuum system was essential to high reproducibility of etching results. According to our experience, the etching rate is sensitive to the detailed configuration of the vacuum furnace 
and may vary in different systems. The vacuum level in the furnace was monitored by a Millipore CML series 0-100Torr vacuum gauge. We used Praxair ultra high purity grade Ar, $\mathrm{H}_{2}$ and $10 \% \mathrm{NH}_{3}$ in $\mathrm{Ar}$ and research grade $\mathrm{O}_{2}$ in our experiments. To adjust the pressure of the gases, we first close all the gas valves and record the base pressure of the system. Then we opened one gas at a time and adjusted the pressure by manual valves. After the pressure reached the target and remained stable for $\sim 1$ minute, we closed the valve and moved on to the next gas. Finally we opened all the gas valves and started heating to a desired temperature. Since we adjusted the pressure manually, there might be some slight pressure variations for different batches, which could cause slight variations in etching rates.

\section{Fabrication and electrical characterization of GNR devices}

After making (or narrowing) GNRs on the $\sim 300 \mathrm{~nm} \mathrm{SiO}_{2} / \mathrm{p}^{++} \mathrm{Si}$ substrate with prepatterned Ti/Au markers, we used AFM to locate the GNR or GNR array relative to a marker. We then used EBL to define source and drain, followed by $\sim 20 \mathrm{~nm}$ Pd evaporation and liftoff. The devices were annealed in $\mathrm{Ar}$ at $\sim 200^{\circ} \mathrm{C}$ to anneal metal contacts. The electrical data of GNR devices were taken by a standard semiconductor analyzer (Agilent 4156C) inside a Lakeshore table-top cryogenic vacuum probe station connected to a turbo pump. The base pressure of the system was $\sim 10^{-6}$ Torr.

\section{Acknowledgement}

This work was supported by Intel, MARCO MSD center, Graphene MURI supported by Office of Naval Research. We gratefully thank James W. Conway in Stanford Nanofabrication Facility for helpful discussions. 


\section{Author contributions}

X. W. and H. D. conceived and designed the experiments. X. W. performed the experiments and analyzed the data. X. W. and H. D. co-wrote the paper. All authors discussed the results and commented on the manuscript. 


\section{Figure captions:}

Figure 1. Gas phase chemical etching and narrowing of graphene sheets. The etching condition used was $\sim 25 \mathrm{mT}$ Torr of $\mathrm{O}_{2}$ in the presence of $\sim 1$ Torr of $10 \% \mathrm{NH}_{3}$ in $\mathrm{Ar}$ at $800^{\circ} \mathrm{C}$ for 1 hour. (a) \& (b) show the same graphene sheet imaged by AFM as made (a) and after etching (b). (c) shows the overlay images before (yellow) and after (red) etching, with uniform etching from the edges observed at an etching rate of $\sim 0.5 \mathrm{~nm} / \mathrm{min}$. (d)-(f) show another set of graphene sheet etching data. A wrinkle with high strain (bright strip) on the asmade graphene was etched away to form a trench. Etching rate was $\sim 0.8 \mathrm{~nm} / \mathrm{min}$. Note that new wrinkles (bright lines in (e)) were observed after the reaction, probably caused by thermal effects. (g) \& (h) Raman G band mapping of the same graphene sheet as in (d) and (e) before and after etching, respectively. (i) Averaged Raman spectra from the graphene plane in (g) and (h). The 2D band can be fit into 4 Lorentzians for both as-made and after etching cases, indicating 2-layer graphene for both before and after etching. ${ }^{21}$ No obvious D band was observed after etching in the plane of graphene.

Figure 2. Lithographically patterned GNR arrays and junctions. (a) Schematics of the fabrications process. (b) An AFM image of a w 20nm GNR array at $\sim 200 \mathrm{~nm}$ pitch. (c) A high resolution AFM image of a $w \sim 22 \mathrm{~nm}$ GNR array at $\sim 500 \mathrm{~nm}$ pitch. (d)-(g) AFM images of various GNR structures including alphabetic characters and zigzag junctions.

Figure 3. Gas phase chemical narrowing of GNRs. (a) \& (b) AFM images of the same GNR array as made (a) and after chemical narrowing (b), respectively. The narrowing condition was $\sim 25 \mathrm{mT}$ Torr of $\mathrm{O}_{2}$ in the presence of $\sim 1$ Torr of $10 \% \mathrm{NH}_{3}$ in $\mathrm{Ar}$ at $800^{\circ} \mathrm{C}$ for $10 \mathrm{mins}$. The GNRs were narrowed uniformly from $\sim 20 \mathrm{~nm}$ to $\sim 8 \mathrm{~nm}$ with no obvious breaks. The etching rate was $\sim 0.6 \mathrm{~nm} / \mathrm{min}$. (c) \& (d) Averaged Raman spectra of the same GNR array in (a) and (b), respectively.

Figure 4. Field-effect transistors from lithographically patterned and chemically narrowed GNRs and parallel GNR arrays. (a) AFM images of a w 8nm GNR array FET (top panel, the 
same array as in Fig. 3b) with inter-digitized electrodes to contact $\sim 40$ GNRs in parallel (channel length $\sim 160 \mathrm{~nm}$ ). Bottom panel is a zoom-in image of the device. Source (S) and drain (D) contacts are marked on both images. (b) Room temperature $I_{d s}-V_{g s}$ characteristics of the GNR array FET in (a) probed in air. The $I_{o n} / I_{\text {off }}$ ratio was $\sim 50$. (c) $I_{d s}-V_{d s}$ characteristics of the same device in (a) probed in air. From top to bottom, $V_{g s}=-40 \mathrm{~V}$ to $50 \mathrm{~V}$ in $10 \mathrm{~V}$ steps. (d) AFM of an as-made and narrowed GNR with breaks along the ribbon. The highlighted part was sub-5nm in width, which was used to make a GNR-FET. (e) Room temperature $I_{d s}-V_{g s}$ characteristics of the GNR-FET fabricated on the GNR in (d). The device showed an $I_{o n} / I_{\text {off }}$ ratio of higher than $10^{4}$ probed in air and in vacuum. $V_{d s}=10 \mathrm{mV}$ for both curves. (f) $I_{d s}-V_{d s}$ characteristics of the same device in (e) probed in vacuum. From top to bottom, $V_{g s}=40 \mathrm{~V}$ to $30 \mathrm{~V}$ in $-10 \mathrm{~V}$ steps. Inset shows the AFM image of the GNR-FET. 


\section{References}

1. Geim, A.K. \& Novoselov, K.S. The rise of graphene. Nature Mater. 6, 183 (2007).

2. Geim, A. K. Graphene: status and prospects. Science 324, 1530 (2009).

3. Li, X. ${ }^{*}$, Wang, X. ${ }^{*}$, Zhang, L., Lee, S. \& Dai, H. Chemically derived, ultrasmooth graphene nanoribbon semidonductors. Science 319, 1229 (2008).

4. Wang, X. et al. Room-temperature all-semiconducting sub-10-nm graphene nanoribbon field-effect transistors. Phys. Rev. Lett. 100, 206803 (2008).

5. Jiao, L., Zhang, L., Wang, X., Diankov, G. \& Dai, H. Narrow graphene nanoribbons from carbon nanotubes. Nature 458, 877 (2009).

6. Bai, J., Duan, X. \& Huang, Y. Rational fabrication of graphene nanoribbons using a nanowire etching mask. Nano Lett. 9, 2083 (2009).

7. Han, M.Y., Ozyilmaz, B., Zhang, Y. \& Kim, P. Energy band-gap engineering of graphene nanoribbons. Phys. Rev. Lett. 98, 206805 (2007).

8. Chen, Z., Lin, Y.-M., Rooks, M.J. \& Avouris, P. Graphene nano-ribbon electronics. Physica E: Low-dimensional Systems and Nanostructures 40, 228 (2007).

9. Pomomarenko, L. A. et al. Chaotic Dirac billiard in graphene quantum dots. Science 320, 356 (2008).

10. Kosynkin, D. V. et al. Longitudinal unzipping of carbon nanotubes to form graphene nanoribbons. Nautre 458, 872 (2009).

11. Nakada, K., Fujita, M., Dresselhaus, G. \& Dresselhaus, M.S. Edge state in graphene ribbons: nanometer size effect and edge shape dependence. Phys. Rev. B 54, 17954 (1996).

12. Son, Y.-W., Cohen, M.L. \& Louie, S.G. Energy gaps in graphene nanoribbons. Phys. Rev. Lett. 97, 216803 (2006).

13. Barone, V., Hod, O. \& Scuseria G.E. Electronic structure and stability of semiconducting graphene nanoribbons. Nano Lett. 6, 2748 (2006).

14. Wang, X., Tabakman, S.M. \& Dai, H. Atomic layer deposition of metal oxides on pristine and functionalized graphene. J. Am. Chem. Soc. 130, 8152 (2008).

15. Lee, S.M., Lee, Y.H., Hwang, Y.G., Hahn, J.R. \& Kang, H. Defected-induced oxidation of graphite. Phys. Rev. Lett. 82, 217 (1999).

16. Hahn, J. R., Kang, H., Lee, S.M. \& Lee, Y.H. Mechanistic study of defect-induced oxidation of graphite. J. Phys. Chem. B 103, 9944 (1999).

17. Ni, Z. H. et al. Uniaxial strain on graphene: Raman spectroscopy study and band-gap opening, ACS Nano 2, 2301 (2008).

18. Mohiuddin, T. M. G. et al. Uniaxial strain in graphene by Raman spectroscopy: G peak splitting, Gruneisen parameters, and sample orientation. Phys. Rev. B 79, 205433 (2009).

19. Li, X. et al. Simultaneous nitrogen-doping and reduction of graphene oxide. J. Am, Chem. Soc. 131, 15939 (2009).

20. Yan, Q. et al. Intrinsic current-voltage characteristics of graphene nanoribbon transistors and effect of edge doping. Nano Lett. 7, 1469 (2007).

21. OuYang, F., Xiao, J., Guo, R, Zhang, H. \& Xu, H. Transport properties of T-shaped and cross junctions based on graphene nanoribbons. Nanotechnology 20, 055202 (2009).

22. Ferrari, A.C. Raman spectroscopy of graphene and graphite: disorder, electronphonon coupling, doping and nonadiabatic effects. Solid State Comm. 143, 47 (2007). 
23. Wang, X. et al. N-doping of graphene through electrothermal reactions with ammonia. Science 324, 768 (2009).

24. Zhao, P., Choudhury, M., Mohanram, K. \& Guo, J. Computational model of edge effects in graphene nanoribbon transistors. Nano Res. 1, 395 (2008).

25. Yoon, Y.\& Guo, J. Effect of edge roughness in graphene nanoribbon transistors. Appl. Phys. Lett. 91, 073103 (2007).

26. Cresti, A. et al. Charge transport in disordered graphene-based low dimensional materials. Nano Res. 1, 361 (2008).

27. Ci, L. et al. Controlled nanocutting of graphene. Nano Res. 1, 116 (2008).

28. Campos, L.C., Manfrinato, V.R., Sanchez-Yamagishi, J.D., Kong, J. \& JarilloHerrero, P. Anisotropic etching and nanoribbon formation in single-layer graphene. Nano Lett. 9, 2600 (2009).

29. Datta, S.S., Strachan, D.R., Khamis, S.M. \& Johnson, A.T.C. Crystallographic etching of few-layer graphene. Nano Lett. 8, 1912 (2008).

30. Nemes-Incze, P., Magda, G., Kamaras, K. \& Biro, L.P. Crystallographically selective nanopatterning of graphene on $\mathrm{SiO}_{2}$. Nano Res. 3, 110 (2010).

31. Novoselov, K. S. et al. Electric field effect in atomically thin carbon films. Science 306, 666 (2004).

32. Hu, W., Sarveswaran, K, Lieberman, M. \& Bernstein, G.H. Sub-10nm electron beam lithography using cold development of ploy(methylmethacrylate). J. Vac. Sci. Tech. B 22, 1711 (2004). 

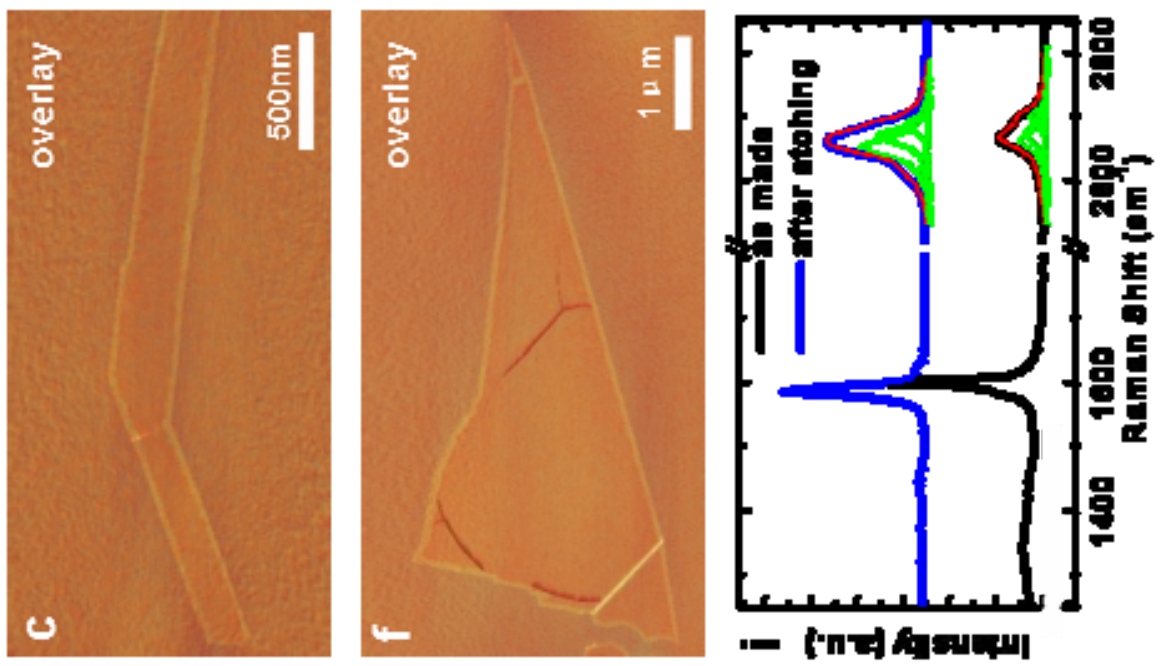

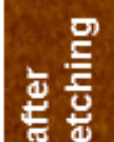
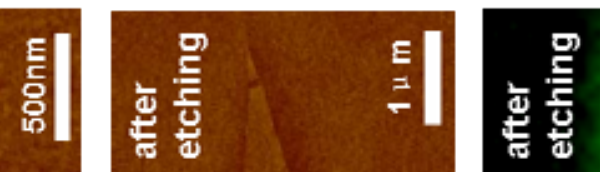

它

c.
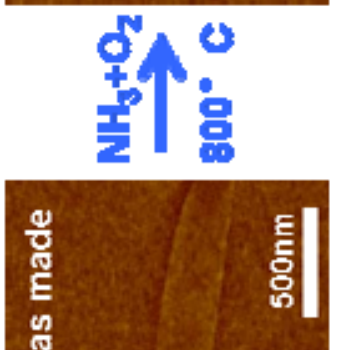

co
逮

๘
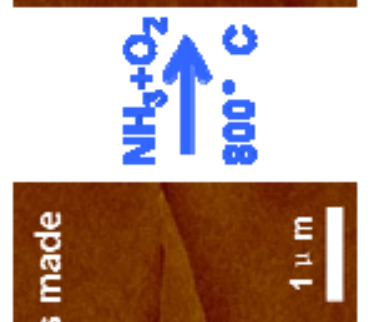

q

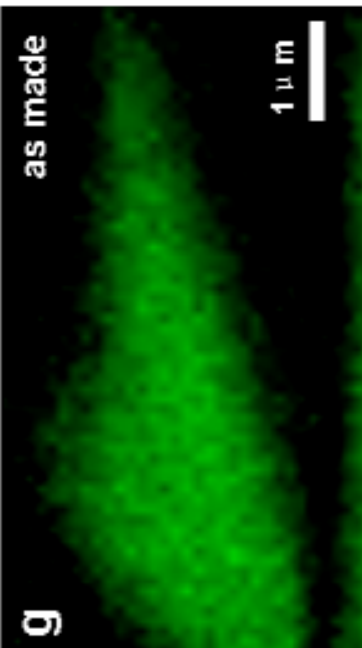



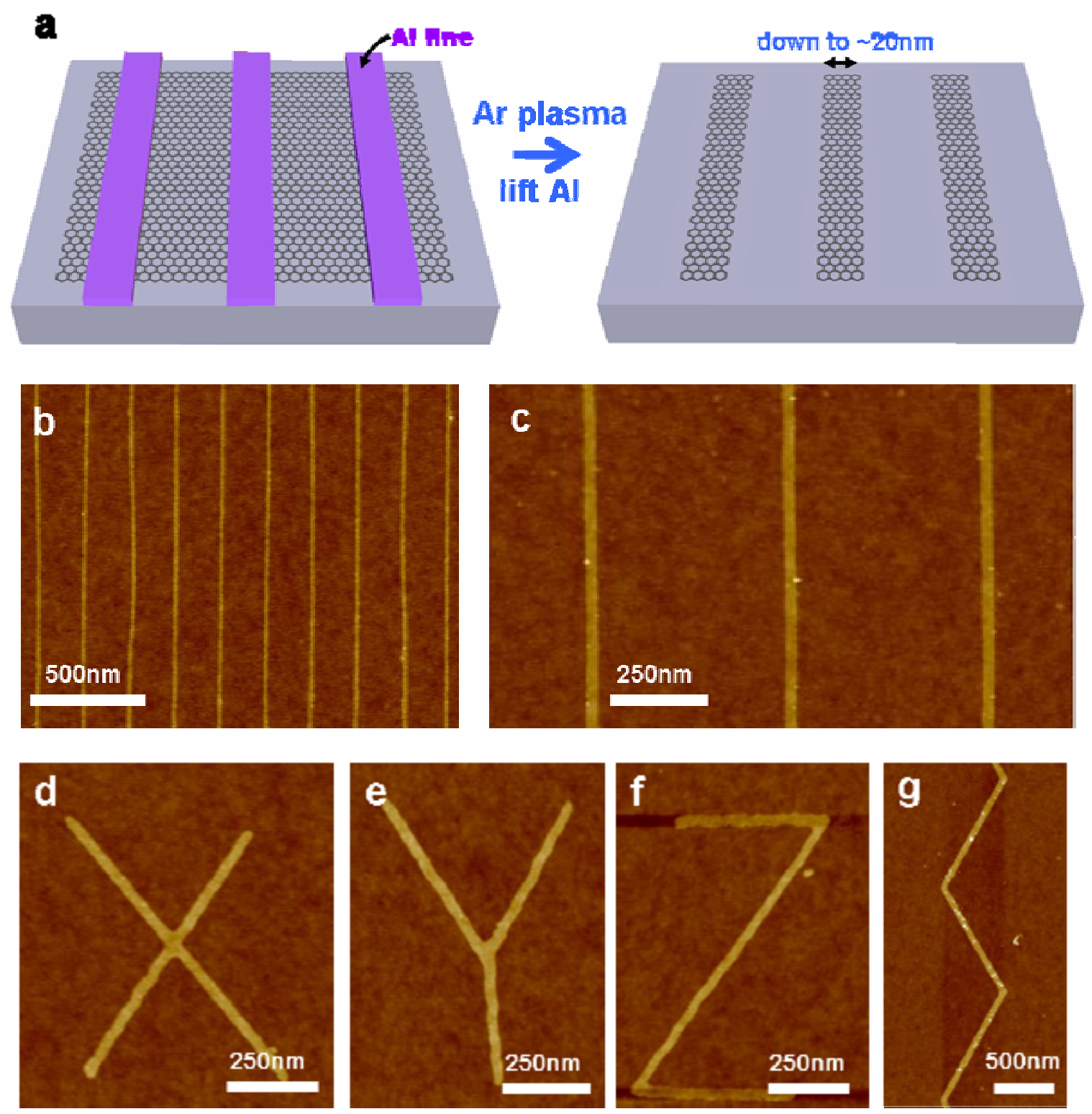

Figure 2 

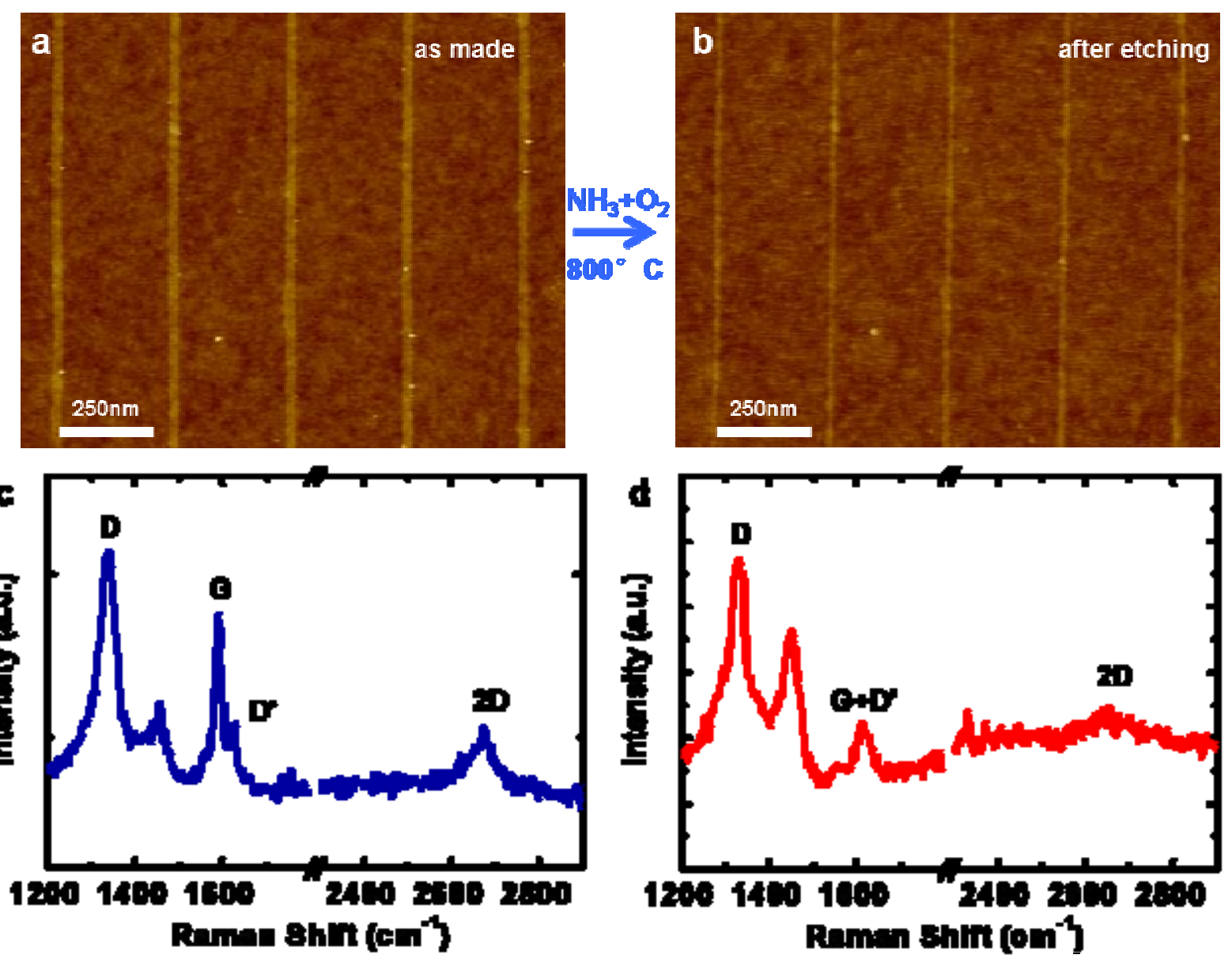

Figure 3 

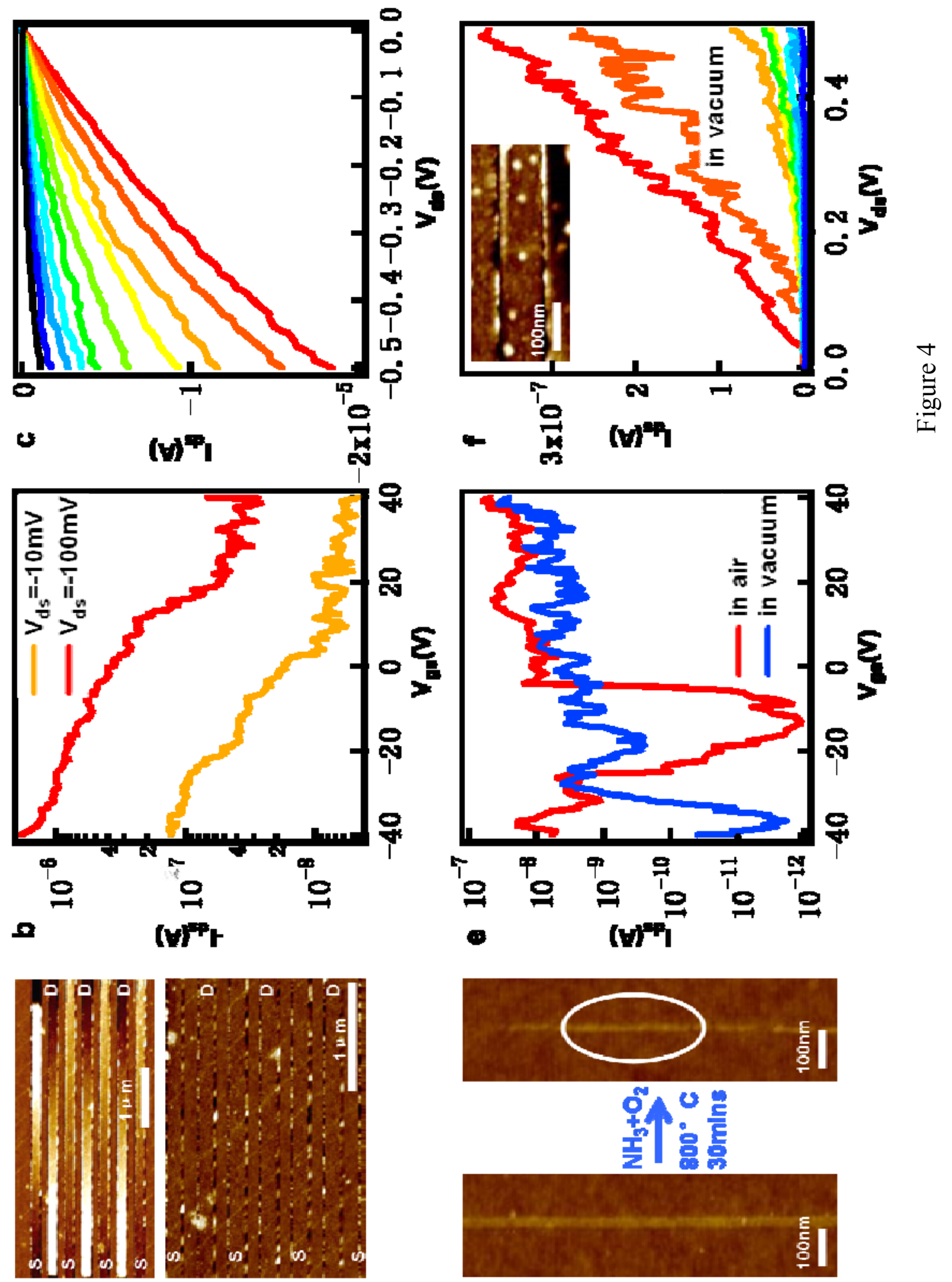